\title{
Therapeutic Effects of Hydrogen in Animal Models of Parkinson's Disease
}

\author{
Kyota Fujita, ${ }^{1}$ Yusaku Nakabeppu, ${ }^{2}$ and Mami Noda ${ }^{1}$ \\ ${ }^{1}$ Laboratory of Pathophysiology, Graduate School of Pharmaceutical Sciences, Kyushu University, 3-1-1 Maidashi, \\ Fukuoka 812-8582, Japan \\ ${ }^{2}$ Division of Neurofunctional Genomics, Medical Institute of Bioregulation, Kyushu University, \\ Fukuoka 812-8582, Japan
}

Correspondence should be addressed to Mami Noda, noda@phar.kyushu-u.ac.jp

Received 15 September 2010; Revised 5 January 2011; Accepted 14 March 2011

Academic Editor: David S. Park

Copyright () 2011 Kyota Fujita et al. This is an open access article distributed under the Creative Commons Attribution License, which permits unrestricted use, distribution, and reproduction in any medium, provided the original work is properly cited.

Since the first description of Parkinson's disease (PD) nearly two centuries ago, a number of studies have revealed the clinical symptoms, pathology, and therapeutic approaches to overcome this intractable neurodegenerative disease. 1-methy-4-phenyl-1,2,3,6tetrahydropyridine (MPTP) and 6-hydroxydopamine (6-OHDA) are neurotoxins which produce Parkinsonian pathology. From the animal studies using these neurotoxins, it has become well established that oxidative stress is a primary cause of, and essential for, cellular apoptosis in dopaminergic neurons. Here, we describe the mechanism whereby oxidative stress evokes irreversible cell death, and propose a novel therapeutic strategy for PD using molecular hydrogen. Hydrogen has an ability to reduce oxidative damage and ameliorate the loss of nigrostriatal dopaminergic neuronal pathway in two experimental animal models. Thus, it is strongly suggested that hydrogen might provide a great advantage to prevent or minimize the onset and progression of PD.

\section{Introduction}

The central pathological feature of PD was loss of neurons in substantia nigra pars compacta $(\mathrm{SNpc})$. DA depletion by the loss of dopaminergic neurons in SNpc is a primary symptom of $\mathrm{PD}[1]$. PD is one of the most common neurodegenerative and progressive diseases, along with Alzheimer's disease (AD) $[2,3]$. In these last two decades, many lines of evidence have emerged to suggest that oxidative stress is closely related to the onset and the progression of $\mathrm{PD}$ and $\mathrm{AD}$.

Using neurotoxins in experimental animal models, an enormous number of studies have been undertaken to develop neuroprotective drugs against PD. MPTP (1-methyl4-phenyl-1,2,3,6-tetrahydropyridine) was found to be a byproduct of the chemical synthesis of a meperidine analog with potent heroin-like effects $[4,5]$. MPTP has the ability to induce PD-like pathology and has been used in various species including nonhuman primates, and rodents. Among the neurotoxic mechanism of MPTP, mitochondrial impairment is highly associated with oxidative damage and related neurodegeneration; the detailed mechanism and the linkage between oxidative damage and neurodegeneration are discussed in this review. Although MPTP-induced PD model animals are regarded as the best reproducible model, another neurotoxin, 6-hydroxydopamine (6-OHDA; 2,4,5trihydroxyphenylethylamine), is also used for toxin-induced animal model of PD [6].

Many trials have focused on the reduction of oxidative stress as a therapeutic strategy because oxidative stress is regarded as one of the major risk factors in the onset of PD as mentioned above. However, there are still no known antioxidant drugs which are clinically used to prevent PD. Here, the neurotoxic mechanism of MPTP which induces Parkinsonian pathology and behavior, and how molecular hydrogen prevents them, is discussed in this review.

\section{Acute and Chronic PD Model Induced by MPTP}

MPTP is a protoxin which is high lipophilic molecule, and can penetrate the blood-brain barrier (BBB) after systemic administration. After crossing the BBB, MPTP is readily 
converted to 1-methyl-4-phenylpyridinium ion $\left(\mathrm{MPP}^{+}\right)$, an actual toxin which can lead to dopaminergic neurodegeneration [7]. MPTP conversion to $\mathrm{MPP}^{+}$is dependent on the activity of monoamine oxidase $\mathrm{B}$ (MAO-B) by a twostep reaction. First, MPTP is converted to the intermediate 1-methyl-4-phenyl-2,3-dihydropyridinium $\left(\mathrm{MPDP}^{+}\right)$, catalyzed by MAO-B [8]. Then, unstable $\mathrm{MPDP}^{+}$dissociates to $\mathrm{MPP}^{+}$and MPTP $[9,10]$. Conversion of MPTP to $\mathrm{MPDP}^{+}$occurs in glial cells and serotonergic cells, not in dopaminergic cells. Dopaminergic neurons exhibit a highaffinity uptake process of $\mathrm{MPP}^{+}$through the dopamine transporter, which allows the neurotoxin $\mathrm{MPP}^{+}$to cause selective dopaminergic neuronal loss [11]. Inside the neurons, $\mathrm{MPP}^{+}$accumulates in the mitochondrial matrix, whose uptake is driven by mitochondrial transmembrane potential gradient $[12,13] . \mathrm{MPP}^{+}$impairs mitochondrial respiration by inhibiting the multisubunit enzyme complex I of the mitochondrial electron transport chain [14, 15]. Inhibition of complex I causes two early and major events: ATP depletion and the buildup of reactive oxygen species (ROS). Complex I activity appears to be decreased by more than $50 \%$ to induce nonsynaptic mitochondrial ATP depletion. In vitro studies also revealed that mitochondria which are isolated from whole brain require $70 \%$ inhibition of complex I activity for ATP depletion. However, in vivo MPTP administration causes only a transient $20 \%$ reduction of ATP levels in mouse striatum and midbrain [16]. In vitro experiments with synaptic mitochondria show that exceeding a threshold of $25 \%$ inhibition of complex I results in significant ATP depletion [17]. These findings may imply that synaptic mitochondria show a better correlation with both complex I inhibition and ATP depletion than those in somatic mitochondria. This may answer the question why dopaminergic neurodegeneration shows retrograde degeneration from striatal nerve terminals, which are rich in synaptic mitochondria.

The extents of loss of dopaminergic neurons and behavioral alteration vary depending on differences in the protocol of MPTP administration. Acute administration $(20 \mathrm{mg} / \mathrm{kg}$, 3 or 4 times at 2 hours interval) can reduce $\sim 70 \%$ of nigral dopaminergic neurons and $\sim 90 \%$ of striatal nerve terminal fibers 7 days after administration when the loss of nigral neurons is stable [18] (see Table 1). Up to $10 \%$ of MPTPadministered involuntarily die within 24 hours because of cardiovascular side effects, not of dopaminergic neuronal loss, and mice were immobilized until 24 to 48 hours. Striatal $\mathrm{MPP}^{+}$level was increased and peaked $\sim 3$ hours after the last administration of MPTP. In subacute injection model, MPTP is administrated once a day at $30 \mathrm{mg} / \mathrm{kg}$ for 5 consecutive days. Since mild doses of MPTP was administered compared to the acute injection model, an incidence of death was lower in the subacute injection model. Loss of nigral dopaminergic neurons and striatal nerve terminal fibers was also less than acute injection model; $\sim 50 \%$ loss of fibers and $\sim 40 \%$ loss of nigral dopaminergic neurons were observed 3 weeks after the last day of MPTP administration. For the continuous administration model, MPTP was infused subcutaneously (s.c.) or intraperitoneally (i.p) using osmotic pumps. Our observation revealed that subcutaneous infusion of MPTP at $45 \mathrm{mg} / \mathrm{kg} /$ day for 28 days caused $50 \%$ loss of nigral dopaminergic neurons [19]. Continuous administration of MPTP subacutely or chronically caused less dopaminergic neuronal loss, which might reflect sprouting of residual fibers or de novo appearance of tyrosine hydroxylase-(TH-) positive dopaminergic neurons in DA-depleted striatum [22-25]. Therefore, chronic recovery and damage of TH fiber may occur simultaneously in nigrostriatal pathway.

The chronic administration model had several unique features which were regarded as better phenomena as PD model: (i) formation of inclusion bodies which were positive for alpha-synuclein and ubiquitin, (ii) loss of noradrenergic (NE) neurons in locus coeruleus, (iii) impairment of ubiquitin-proteasome system, and (iv) behavioral alteration. Especially, loss of NE neurons was observed as in human PD [26], and dopamine $\beta$-hydroxylase knockout $\left(D b h^{-/-}\right)$mice which lack NE neurons showed more profound motor deficit compared to MPTP-treated mice [27]. Furthermore, bolus administration of MPTP did not induce inclusion bodies formation [21]. Therefore, chronic administration model using an osmotic pump could mimic human PD feature.

\section{Oxidative Damage and Apoptotic Signals in MPTP Model}

ROS, mostly a superoxide, is produced in mitochondria because of a leak of electrons from the respiratory chain inhibited by $\mathrm{MPP}^{+}$[28]. Energy metabolic inhibition and ROS overproduction have their peak several hours after MPTP administration, which trigger the downstream of cellular apoptosis and neurodegeneration days after MPTP treatment $[29,30]$. In PD patients, iron level is increased selectively in $\mathrm{SNpc}$, which leads to the greater accessibility of ferrous iron $\left(\mathrm{Fe}^{2+}\right)$ with hydrogen peroxide and thus generating hydroxyl radical $(\bullet \mathrm{OH})[31]$. Moreover, lipid peroxidation, protein carbonyls, and 8-oxo-7,8-dihydroguanine (8-oxoG) are increased, which means that cellular lipids, proteins, and DNA are highly exposed to oxidative stress $[32,33]$. Such oxidative damage occurs prior to the cellular apoptosis processes.

Sources of ROS are various, and ROS is produced not only in neurons but also in glial cells such as microglia when they become activated (reactive) and show morphological changes [34]. From dopaminergic neurons, superoxide is produced not only in mitochondria but also by autooxidation of DA [35]. It is known that auto-oxidation of DA leads to the production of DA (semi)quinones that are converted into aminochrome, which can generate superoxide $[36,37]$. Increased ROS causes oxidative damage to DNA [38, 39], cellular lipid peroxidation [40,41], and stress-related signaling activation such as MAPK and JNK activation [42-44].

Oxidative stress in DNA leads to cellular apoptosis which is mediated by p53 activation and p53-derived Bax translocation to mitochondria. Furthermore, Bax translocation and cytochrome $\mathrm{c}$ from mitochondria to the cytosol leads to caspase-dependent apoptosis [45]. Oxidative damage in DNA induces not only caspase-dependent apoptosis but also caspase-independent apoptosis. Among the five normal 
TABLE 1: Comparison of representative MPTP-PD models. Each written model is representative and reproducible examples of MPTP-PD model because many researchers modify their own protocols in creating MPTP-PD model.

\begin{tabular}{|c|c|c|c|}
\hline & Acute & Sub-acute & Chronic \\
\hline Dose of MPTP & $20 \mathrm{mg} / \mathrm{kg}$ & $30 \mathrm{mg} / \mathrm{kg}$ & $\begin{array}{c}30 \text { or } 45 \mathrm{mg} / \mathrm{kg} / \mathrm{day} \\
\text { (using osmotic pump) }\end{array}$ \\
\hline Duration of MPTP & 3 or 4 times at $2 \mathrm{~h}$ interval & Once a day for 5 consecutive days & 28 days \\
\hline Administration of MPTP & i.p. & i.p. & $\begin{array}{l}\text { i.p. }(30 \mathrm{mg}) \\
\text { s.c. }(45 \mathrm{mg})\end{array}$ \\
\hline Extirpation of brain & 7 days after injection & 21 days after injection & 28 days after pump infusion \\
\hline Anticipating nigral cell loss & $70 \%$ & $40 \%$ & $50 \%$ \\
\hline Anticipating striatal fiber loss & $90 \%$ & $50 \%$ & $50 \%$ \\
\hline Notable features & Undesirable death $(\sim 10 \%)$ & $\begin{array}{l}\text { Less or no undesirable death } \\
\text { Nitrated } \alpha \text {-synuclein accumulation }\end{array}$ & $\begin{array}{c}\text { Behavioral alteration } \\
\text { (open-field test) } \\
\text { Formation of inclusion bodies } \\
\text { (stained for } \alpha \text {-synuclein, ubiquitin) } \\
\text { Loss of noradrenergic neurons }\end{array}$ \\
\hline References & {$[18,19]$} & {$[18,20]$} & {$[18,19,21]$} \\
\hline
\end{tabular}

nucleobases, guanine is the most susceptible to oxidation, and the C8 position of free deoxyguanosine $(\mathrm{dG})$ or dGTP is the most effectively oxidized by $\bullet \mathrm{OH}$ in comparison to those in DNA. In fact, eight- to nine-times more 8-oxoG is formed in nucleotide dGTP than in DNA [46, 47]. Under the oxidative stress condition, 8-oxoG accumulates in mitochondrial and nuclear DNA, which can be selectively visualized by immunohistological technique [39, 48]. Systemic MPTP administration promoted the accumulation of 8oxoG both in mitochondria DNA and in nuclear DNA [39]. Mitochondrial 8-oxoG (mt8oxoG) accumulated in nerve terminal in the striatum, prior to nuclear 8-oxoG (nu8oxoG) accumulation in nigral dopaminergic neurons. Oka et al. [49] demonstrated that accumulation of mt8oxoG causes mitochondrial dysfunction resulting in ATP depletion, which can open the mitochondrial membrane permeability transition (MMPT) pore. During replication of mitochondrial DNA (mtDNA) with an increased level of 8-oxoG, adenine is frequently inserted opposite 8-oxoG in mtDNA, and such adenine paired with 8 -oxoG is selectively excised by adenine DNA glycosylase encoded by Mutyh gene. During the base excision repair (BER) process, apurinic/apyrimidinic (AP) endonuclease or AP lyases convert abasic sites to singlestrand breaks (SSBs) [50-53]. It has been demonstrated that the MUTYH-initiated BER causes mtDNA degradation resulting in its depletion under oxidative stress [49]. This depletion may induce a decreased supply of mitochondriaencoded proteins, transfer RNAs, and ribosomal RNAs, leading to dysfunction of mitochondrial respiration. Therefore, accumulation of mt8oxoG results in the depletion of ATP. Furthermore, MMPT opening enables $\mathrm{Ca}^{2+}$ to leave mitochondria, and cytoplasmic $\mathrm{Ca}^{2+}$ increase activates calpain, a ubiquitous calcium-sensitive protease, thus inducing cell death $[49,54]$. It has been well documented that calpain activation causes the cleavage of neuronal substrates that negatively affect neuronal structure and function, leading to inhibition of essential mechanisms for neuronal survival [55]. Moreover, inhibition of calpain is known to reduce the dopaminergic neuronal loss in the MPTP model [56]. Taken together, we propose that oxidative stress in dopamine neurons initiated by MPTP administration increases accumulation of mt8oxoG, and thereby causes mitochondrial dysfunction resulting in dopaminergic neuronal loss which is dependent on the calpain pathway (Figure 1).

On the other hands, SSBs are accumulated in nuclear DNA as a result of excision of adenine opposite nu8oxoG by MUTYH, and activate poly (ADP-ribose) polymerase (PARP) with the increase of poly-ADP ribosylation, leading to nuclear translocation of apoptosis inducing factor (AIF) and NAD/ATP depletion [49]. PARP, known as a molecular nick-sensor, binds SSBs specifically and utilizes $\beta$-NAD ${ }^{+}$ as a substrates to catalyze the synthesis of (ADP-ribose) polymers (poly-ADP ribosylation) on nuclear proteins, including PARP itself with the increase of PARP activity $[57,58]$. PARP activation signal induces AIF release from mitochondria and translocation to the nucleus, which results in a caspase-independent pathway of programmed cell death [59]. Activation of PARP leads to its autoconsumption, and depletes ATP content. Therefore, a loss of energy supply also contributes to cell death [49]. Several reports indicate that PARP activation is associated with MPTP-derived neurotoxicity $[60,61]$. It is, however, noteworthy that MUTYH-dependent PARP activation requires replication of nuclear DNA [49], indicating that mitotic cells in brain such as glial cells other than neurons may be affected by the PARP-AIF pathway with increased level of nu8oxoG. Among glial cells, oligodendrocytes and astrocytes show PARP-AIF pathway mediated apoptotic cell death $[62,63]$. Therefore, 


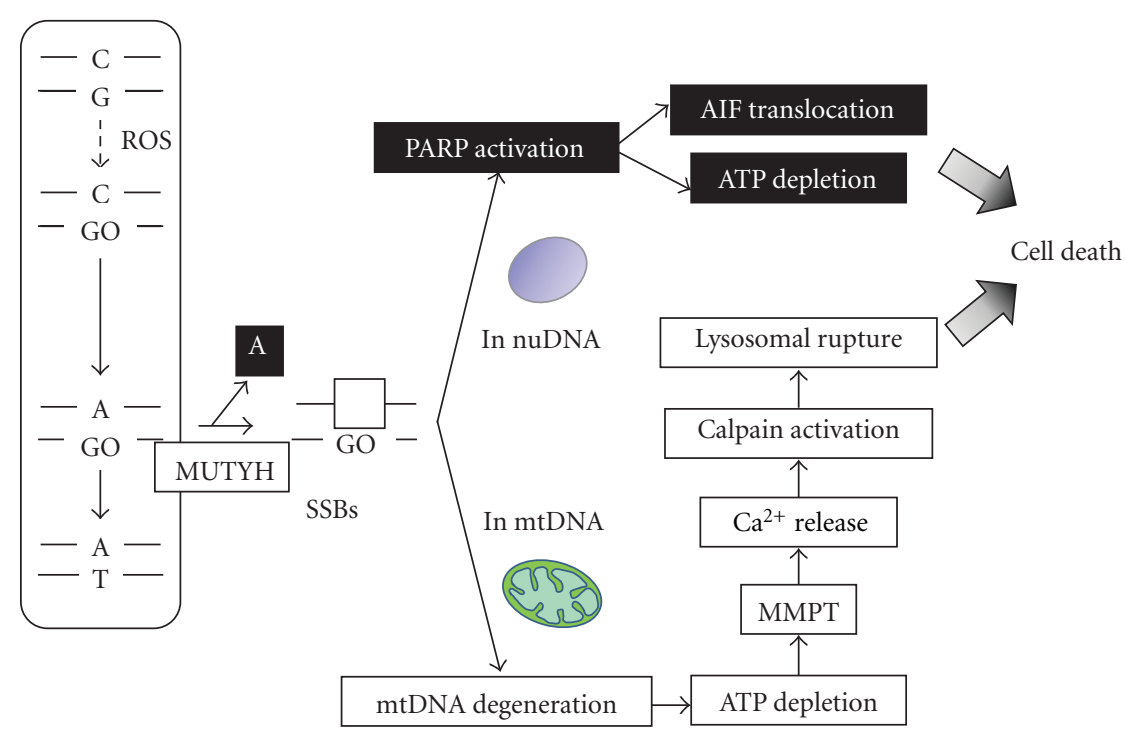

FIGURE 1: Scheme of apoptotic death signaling by accumulation of 8-oxoguanine (8-oxoG; GO) and single-strand-breaks (SSBs) in DNA. ROS, especially hydroxyl radical, increase the 8-oxoG accumulation and SSBs by MUTYH. In the case of SSBs in nucleus, activation of poly (ADP-ribose) polymerase (PARP), apoptosis inducing factor (AIF) translocation from mitochondria to nucleus, and ATP depletion followed by $\mathrm{NAD}^{+}$depletion leads to cellular apoptosis. On the other hands, in mitochondria, accumulation of SSBs induces mitochondrial DNA (mtDNA) degeneration. Loss of function of energy supply leads to ATP depletion, and mitochondrial membrane permeability transition (MMPT), and calpain activation results in lysosomal rupture, which potentiates cell death (modified from Figure 8, Oka et al., 2008 [49]).

accumulation of 8-oxoG in nuDNA in glial cells may thus cause caspase-independent cellular apoptosis, which might play critical roles in neurodegeneration (Figure 1).

\section{6-OHDA Model and Oxidative Damage in Nigrostriatal Neurons}

For PD model animal, 6-OHDA is also used for deletion of catecholamine in the brain and in periphery [64]. 6-OHDA serves as a neurotoxin; which is readily auto-oxidized and deaminated by monoamine oxidase (MAO) [65]. Because 6OHDA cannot penetrate blood-brain barrier, direct administration into the brain is required for the neurodegeneration in 6-OHDA model [66]. This neurotoxin can be generated within the brain by nonenzymatic reaction of dopamine, hydrogen peroxide, and free iron [67-69]. Auto-oxidation of dopamine by nitrite ions or manganese can also generate 6-OHDA [70, 71]. Oxidative damage via hydrogen peroxide and derived $\bullet \mathrm{OH}$ are associated with the neurotoxic mechanism by 6-OHDA [64]. The steps to generate ROS are several varied processes: (1) in physiological condition, 6-OHDA is subjected to non-enzymatic auto-oxidation and generates several toxic products such as quinones, superoxide anion radicals, hydrogen peroxides, and $\bullet \mathrm{OH}$ [65]; (2) Fenton reaction initiates and/or amplifies ROS generation. The deamination by MAO, or auto-oxidization increases the hydrogen peroxide $[72,73]$. Both neurotoxins, MPTP and 6-OHDA, can potentiate the cellular apoptosis with the increase of oxidative damage in DNA, but SSBs-derived PARP activation does not affect 6-OHDA-derived cell death in embryonic nigral grafts [74]. This might be because of less formation of NO in grafted nigral neurons [75].
The apoptotic mechanism by 6-OHDA is explained by the role of p53 and Bax translocation, and caspase activation [66].

\section{Hydrogen as a Therapeutic Antioxidant for Experimental Animal Models of PD}

Since the first striking evidence indicating that molecular hydrogen acts as an antioxidant and inhalation of hydrogencontaining gas reduces ischemic injury in brain [76], there have been increasing reports which support therapeutic properties of hydrogen against oxidative stress-related diseases and damages in brain [77, 78], liver [79], intestinal graft [80], myocardial injury [81, 82], and atherosclerosis [83]. Hydrogen can be taken up by inhalation of hydrogencontaining gas (hydrogen gas) or drinking hydrogencontaining water (hydrogen water). One hour after the start of inhalation of hydrogen gas, hydrogen can be detectable in blood, at levels of $10 \mu \mathrm{M}$ in arterial blood [76]. The content of hydrogen can be measured even after intake of hydrogen water by a catheter, which shows $5 \mu \mathrm{M}$ in artery calculated after $3 \mathrm{~min}$ of hydrogen water incorporation [77]. Taking into account its continuous intake, it is easier and safer to drink hydrogen water than inhaling hydrogen gas.

We have previously reported that hydrogen in drinking water reduced the loss of dopaminergic neurons in MPTPtreated mice [19]. The therapeutic effects of hydrogen water against PD model have also been confirmed in another animal model, 6-OHDA-treated rats [84]. It is reported that 6-OHDA also causes 8-oxoG accumulation and mitochondrial dysfunction through oxidative stress [85], and thus our model shown in Figure 1 can be applied to the PD model. 


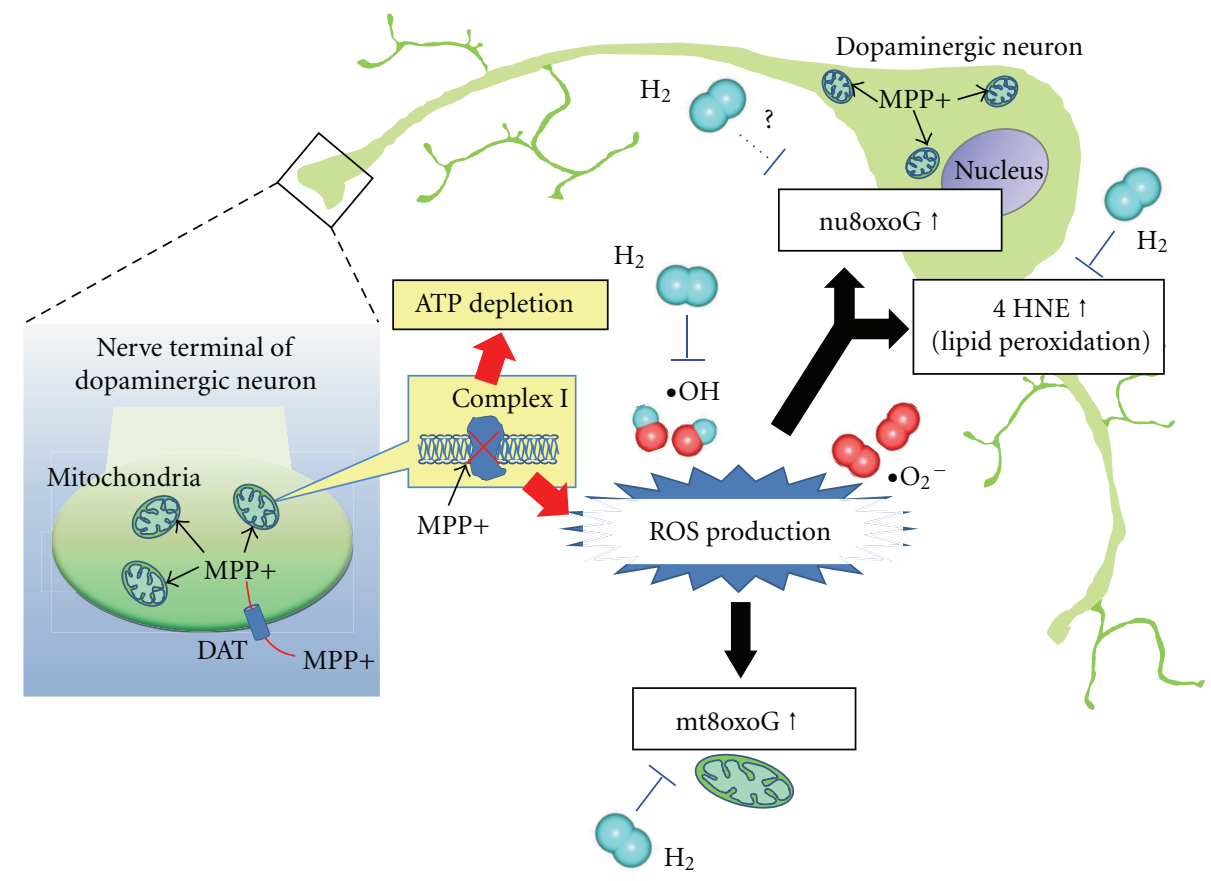

FIGURE 2: The effects of hydrogen in oxidative stress-derived neural apoptosis in dopaminergic cells. Hydrogen $\left(\mathrm{H}_{2}\right)$ selectively reduces hydroxyl radical $(\bullet \mathrm{OH})$ by direct reaction, and decreased oxidative damage such as mitochondrial/nuclear 8 -oxoG (mt8oxoG/nu8oxoG) accumulation, and 4-hydroxynonenal (4-HNE) production in dopaminergic neurons. Each oxidative damage is involved in different neuronal apoptosis. Abbreviation; $\mathrm{MPP}^{+}$: 1-methyl-4-phenylpyridinium ion, DAT: dopamine transporter, ROS: reactive oxygen species, ATP: adenosine $5^{\prime}$-triphosphate, $\bullet \mathrm{OH}$ : hydroxyl radical, $\bullet \mathrm{O}_{2}^{-}$: superoxide, 4 -HNE: 4-hydroxynonenal.

In these animal models, a number of dopaminergic neurons in SNpc, as well as nerve terminal fibers in striatum, were decreased by administration of the neurotoxin. However, hydrogen water significantly reduces the loss of both neuronal cell bodies and fibers compared with normal water. In MPTP-treated mice, chronic administration using an osmotic minipump results in neuronal loss as well as behavioral impairments observed by the open-field test [21]. Rats administered with 6-OHDA also show behavioral impairments assessed by the rotarod test. Hydrogen improved behavioral impairment in both MPTP and 6OHDA model. From these observations, hydrogen water even prevents behavioral alteration which is regarded as a major symptom in PD.

It would provide us with useful information for the design of a therapeutic strategy to investigate how long the neuroprotection acquired by hydrogen water lasts. Continuous intake of hydrogen water before and during MPTP administration showed significant neuroprotection. However, intake of hydrogen water even after MPTP administration also reduced neurotoxic damage [19]. PD is regarded as a progressive neurodegenerative disease, so daily intake of hydrogen water might prevent the disease progression as well as the onset of neurodegeneration.

It has been reported that hydrogen reduced cytotoxic - OH selectively whereas the production of other radicals such as superoxide, hydrogen peroxide and nitric oxide was not altered by hydrogen [76]. This selectivity was proved by cell-free system, and in particular, the preference of scavenging of $\bullet \mathrm{OH}$ rather than superoxide was confirmed in PC12 cell culture system [76]. According to Setsukinai et al. [86], both $\bullet \mathrm{OH}$ and peroxynitrite $\left(\mathrm{ONOO}^{-}\right)$were much more reactive than other ROS. This would be an answer why hydrogen shows selective reaction with only the strongest radicals both in the cell-free system and in PC12 cells.

Especially, $\bullet \mathrm{OH}$ overproduction in oxidative and neurotoxic reaction by MPTP leads to lipid peroxidation observed by 4-hydroxynonenal (4-HNE) immunostaining in nigral dopaminergic neurons prior to cellular death. 4HNE immunoreactivity in MPTP-treated mice is increased by three-times as much as in saline-treated mice [19], which was similar to the previous report of 4-HNE protein levels in substantia nigra observed at the same periods after MPTP administration using HPLC [41]. Hydrogen water significantly reduces the formation of 4-HNE in dopaminergic neurons in the substantia nigra to the level of control [19] (Figure 2). On the other hand, the increase of superoxide, which is detectable by administration of dihydroethidine (DHE) intravenously, was not significantly reduced by hydrogen water [19]. Although hydrogen reduces the production of superoxide in brain slices in hypoxia/reperfusion injury [87], hydrogen water might show a preferential reduction of $\bullet \mathrm{OH}$ during the protection of dopaminergic neurons.

Hydrogen water significantly reduces the accumulation of 8-oxoG in striatum after MPTP administration [19] (Figure 2). As mentioned above, 8-oxoG, an oxidized form of guanine, accumulates both in mitochondria and in 
nucleus; their nomenclature are mt8oxoG and nu8oxoG, respectively. Mt8oxoG accumulates in striatum which are rich in mitochondria in nerve terminal of dopaminergic neurons projected from the substantia nigra. Although nu8oxoG was not detected in nigral cell nucleus [19], hydrogen water might prevent the mt8oxoG-induced cellular apoptotic signals, not just reduce $\bullet \mathrm{OH}$ in dopaminergic nerve terminals.

Hydrogen was effective when it was inhaled during reperfusion; when hydrogen was inhaled just during ischemia (not in reperfusion), infarct volume was not significantly decreased [76]. It was shown that hydrogen in the brain decreased immediately after stopping inhalation and completely disappeared within $10 \mathrm{~min}$ [19], indicating that the effect of hydrogen can be observed only during the period when the oxidative insults occur. Hydrogen could be detected in the blood 3 min after administration of hydrogen water into the stomach [77]. However, unpublished data showed that the half-life of hydrogen in the muscle in rats was approximately $20 \mathrm{~min}$ after the administration of hydrogen gas. Taking these reports into consideration, hydrogen in the brain and other tissues does not stay long enough to exert its ability as an antioxidant to ROS directly. Therefore, it is unlikely that direct reaction of hydrogen itself with ROS plays a major role in the neuroprotection, especially by hydrogen in drinking water, although hydrogen itself has the ability to reduce $\bullet \mathrm{OH}$ preferentially. In accordance with this hypothesis, previous reports from Nakao et al. [88] has demonstrated that drinking hydrogen water increases urinary antioxidant enzyme, superoxide dismutase (SOD), an endogenous defensive system against ROS- (especially superoxide-) mediated cellular damage. Although it takes eight weeks for significant increase of SOD in humans, hydrogen has the ability to alter the expression level of urinary antioxidant enzyme. It was also reported that hydrogen water increased total bilirubin for four to eight weeks compared to baseline. Bilirubin is produced by the catalytic reaction of heme oxygenase 1 (HO-1), and degradation of heme generates bilirubin as well as carbon monoxide and free iron. The increase of HO-1 expression is likely due to the response to oxidative stress, and this response is also characterized as a phase II antioxidant which is positively regulated by several stress-responsive transcriptional factors [89]. Therefore, taking these observations into account, we might better have another aspect for protective effect of hydrogen in drinking water apart from inhalation. It is possible that drinking of hydrogen water has not only the ability to reduce cytotoxic radicals, but also novel mechanisms which are related to anti-oxidative defense system.

\section{Conclusion}

Oxidative stress is a key factor to induce cellular apoptosis in MPTP- and 6-OHDA-derived neurotoxicity. From studies using postmortem human brain of PD patients, increased iron, oxidation of proteins and DNA, lipid peroxidation in the $\mathrm{SN}$ appear to be important findings of oxidative stress [90-93]. Thought there are effective antioxidants or therapeutic strategies for $\mathrm{PD}$, reduction of oxidative stress would be more desirable to attenuate neurotoxic damage in PD. Here, we would like to address that one of the most efficient ways to attenuate oxidative stress is taking low concentration of hydrogen in drinking water, a safer and easier way of hydrogen intake. Although the precise mechanism how hydrogen works is still under investigation, it will be possible to reveal the mechanisms using conventional PD models such as MPTP and 6-OHDA models. Not only that it is of great interest to know the neuroprotective mechanism of hydrogen but also hydrogen will bring great beneficial effects to reduce a risk of lifestyle-related oxidative damage and related neurodegenerative diseases including PD.

\section{Acknowledgment}

The authors thank Professor David. A. Brown (UCL, UK) for reading the paper and providing useful comments.

\section{References}

[1] W. Dauer and S. Przedborski, "Parkinson's disease: mechanisms and models," Neuron, vol. 39, no. 6, pp. 889-909, 2003.

[2] C. K. Glass, K. Saijo, B. Winner, M. C. Marchetto, and F. H. Gage, "Mechanisms Underlying Inflammation in Neurodegeneration," Cell, vol. 140, no. 6, pp. 918-934, 2010.

[3] A. E. Lang and A. M. Lozano, "Parkinson's disease," The New England Journal of Medicine, vol. 339, no. 15, pp. 1044-1053, 1998.

[4] A. Ziering, L. Berger, S. D. Heineman, and J. Lee, "Piperidine derivatives. Part III. 4-arylpiperidines," Journal of Organic Chemistry, vol. 12, no. 6, pp. 894-903, 1947.

[5] S. Przedborski, K. Tieu, C. Perier, and M. Vila, "MPTP as a mitochondrial neurotoxic model of Parkinson's disease," Journal of Bioenergetics and Biomembranes, vol. 36, no. 4, pp. 375-379, 2004.

[6] F. Blandini, M. T. Armentero, and E. Martignoni, "The 6hydroxydopamine model: news from the past," Parkinsonism and Related Disorders, vol. 14, no. 2, pp. S124-S129, 2008.

[7] J. W. Langston, I. Irwin, E. B. Langston, and L. S. Forno, "1-Methyl-4-phenylpyridinium ion (MPP): identification of a metabolite of MPTP, a toxin selective to the substantia nigra," Neuroscience Letters, vol. 48, no. 1, pp. 87-92, 1984.

[8] K. Chiba, A. Trevor, and N. Castagnoli, "Metabolism of the neurotoxic tertiary amine, MPTP, by brain monoamine oxidase," Biochemical and Biophysical Research Communications, vol. 120, no. 2, pp. 574-578, 1984.

[9] K. Chiba, L. A. Peterson, and K. P. Castagnoli, "Studies on the molecular mechanism of bioactivation of the selective nigrostriatal toxin 1-methyl-4-phenyl-1,2,3,6-tetrahydropyridine," Drug Metabolism and Disposition, vol. 13, no. 3, pp. 342-347, 1985.

[10] L. A. Peterson, P. S. Caldera, A. Trevor, K. Chiba, and N. Castagnoli, "Studies on the 1-methyl-4-phenyl-2,3dihydropyridinium Species 2,3-MPDP+, the monoamine oxidase catalyzed oxidation product of the nigrostriatal toxin 1methyl-4-phenyl-1,2,3,6-tetrahydropyridine (MPTP)," Journal of Medicinal Chemistry, vol. 28, no. 10, pp. 1432-1436, 1985.

[11] J. A. Javitch, R. J. D'Amato, S. M. Strittmatter, and S. H. Snyder, "Parkinsonism-inducing neurotoxin, N-methyl-4phenyl-1,2,3,6-tetrahydropyridine: uptake of the metabolite $\mathrm{N}$-methyl-4-phenylpyridine by dopamine neurons explains 
selective toxicity," Proceedings of the National Academy of Sciences of the United States of America, vol. 82, no. 7, pp. 21732177, 1985.

[12] R. R. Ramsay, J. Dadgar, A. Trevor, and T. P. Singer, "Energydriven uptake of N-methyl-4-phenylpyridine by brain mitochondria mediates the neurotoxicity of MPTP," Life Sciences, vol. 39, no. 7, pp. 581-588, 1986.

[13] R. R. Ramsay and T. P. Singer, "Energy-dependent uptake of $\mathrm{N}$-methyl-4-phenylpyridinium, the neurotoxic metabolite of 1-methyl-4-phenyl-1,2,3,6-tetrahydropyridine, by mitochondria," The Journal of Biological Chemistry, vol. 261, no. 17, pp. 7585-7587, 1986.

[14] W. J. Nicklas, I. Vyas, and R. E. Heikkila, "Inhibition of NADH-linked oxidation in brain mitochondria by 1-methyl4-phenyl-pyridine, a metabolite of the neurotoxin, 1-methyl4-phenyl-1,2,5,6-tetrahydropyridine," Life Sciences, vol. 36, no. 26, pp. 2503-2508, 1985.

[15] Y. Mizuno, N. Sone, and T. Saitoh, "Effects of 1-methyl4-phenyl-1,2,3,6-tetrahydropyridine and 1-methyl-4phenylpyridinium ion on activities of the enzymes in the electron transport system in mouse brain," Journal of Neurochemistry, vol. 48, no. 6, pp. 1787-1793, 1987.

[16] P. Chan, L. E. DeLanney, I. Irwin, J. W. Langston, and D. Di Monte, "Rapid ATP loss caused by 1-methyl-4-phenyl-1,2,3,6tetrahydropyridine in mouse brain," Journal of Neurochemistry, vol. 57, no. 1, pp. 348-351, 1991.

[17] G. P. Davey, S. Peuchen, and J. B. Clark, "Energy thresholds in brain mitochondria: potential involvement in neurodegeneration," The Journal of Biological Chemistry, vol. 273, no. 21, pp. 12753-12757, 1998.

[18] V. Jackson-Lewis and S. Przedborski, "Protocol for the MPTP mouse model of Parkinson's disease," Nature Protocols, vol. 2, no. 1, pp. 141-151, 2007.

[19] K. Fujita, T. Seike, N. Yutsudo et al., "Hydrogen in drinking water reduces dopaminergic neuronal loss in the 1-methyl4-phenyl-1,2,3,6-tetrahydropyridine mouse model of Parkinson's disease," PLoS ONE, vol. 4, no. 9, Article ID e7247, 2009.

[20] E. J. Benner, R. Banerjee, A. D. Reynolds et al., "Nitrated $\alpha$-synuclein immunity accelerates degeneration of nigral dopaminergic neurons," PLoS ONE, vol. 3, no. 1, Article ID e1376, 2008.

[21] F. Fornai, O. M. Schlüter, P. Lenzi et al., "Parkinson-like syndrome induced by continuous MPTP infusion: convergent roles of the ubiquitin-proteasome system and $\alpha$-synuclein," Proceedings of the National Academy of Sciences of the United States of America, vol. 102, no. 9, pp. 3413-3418, 2005.

[22] D. D. Song and S. N. Haber, "Striatal responses to partial dopaminergic lesion: evidence for compensatory sprouting," Journal of Neuroscience, vol. 20, no. 13, pp. 5102-5114, 2000.

[23] X. Du, N. D. Stull, and L. Iacovitti, "Brain-derived neurotrophic factor works coordinately with partner molecules to initiate tyrosine hydroxylase expression in striatal neurons," Brain Research, vol. 680, no. 1-2, pp. 229-233, 1995.

[24] J. T. Greenamyre, "Dopaminergic neurons intrinsic to the primate striatum," Journal of Neuroscience, vol. 17, no. 17, pp. 6761-6768, 1997.

[25] G. E. Meredith, "Immunocytochemical characterization of catecholaminergic neurons in the rat striatum following dopamine-depleting lesions," European Journal of Neuroscience, vol. 11, no. 10, pp. 3585-3596, 1999.

[26] M. R. Marien, F. C. Colpaert, and A. C. Rosenquist, "Noradrenergic mechanisms in neurodegenerative diseases: a theory," Brain Research Reviews, vol. 45, no. 1, pp. 38-78, 2004.
[27] K. S. Rommelfanger, G. L. Edwards, K. G. Freeman, L. C. Liles, G. W. Miller, and D. Weinshenker, "Norepinephrine loss produces more profound motor deficits than MPTP treatment in mice," Proceedings of the National Academy of Sciences of the United States of America, vol. 104, no. 34, pp. 13804-13809, 2007.

[28] E. Hasegawa, K. Takeshige, T. Oishi, Y. Murai, and S. Minakami, "1-Methyl-4-phenylpyridinium (MPP) induces NADH-dependent superoxide formation and enhances $\mathrm{NADH}$-dependent lipid peroxidation in bovine heart submitochondrial particles," Biochemical and Biophysical Research Communications, vol. 170, no. 3, pp. 1049-1055, 1990.

[29] E. Fahre, J. Monserrat, A. Herrero, G. Barja, and M. L. Leret, "Effect of MPTP on brain mitochondrial HO and ATP production and on dopamine and DOPAC in the striatum," Journal of Physiology and Biochemistry, vol. 55, no. 4, pp. 325332, 1999.

[30] T. P. Singer, R. R. Ramsay, K. McKeown, A. Trevor, and N. E. Castagnoli, "Mechanism of the neurotoxicity of 1-methyl-4phenylpyridinium (MPP), the toxic bioactivation product of 1-methyl-4-phenyl-1,2,3,6-tetrahydropyridine (MPTP)," Toxicology, vol. 49, no. 1, pp. 17-23, 1988.

[31] J. M. C. Gutteridge, "Iron and oxygen radicals in brain," Annals of Neurology, vol. 32, pp. S16-S21, 1992.

[32] Z. I. Alam, A. Jenner, S. E. Daniel et al., "Oxidative DNA damage in the Parkinsonian brain: an apparent selective increase in 8-hydroxyguanine levels in substantia nigra," Journal of Neurochemistry, vol. 69, no. 3, pp. 1196-1203, 1997.

[33] J. Zhang, G. Perry, M. A. Smith et al., "Parkinson's disease is associated with oxidative damage to cytoplasmic DNA and RNA in substantia nigra neurons," American Journal of Pathology, vol. 154, no. 5, pp. 1423-1429, 1999.

[34] E. C. Hirsch and S. Hunot, "Neuroinflammation in Parkinson's disease: a target for neuroprotection?" The Lancet Neurology, vol. 8, no. 4, pp. 382-397, 2009.

[35] D. G. Graham, "Oxidative pathways for catecholamines in the genesis of neuromelanin and cytotoxic quinones," Molecular Pharmacology, vol. 14, no. 4, pp. 633-643, 1978.

[36] J. Boada, B. Cutillas, T. Roig, J. Bermúdez, and S. Ambrosio, "MPP ${ }^{+}$-induced mitochondrial dysfunction is potentiated by dopamine," Biochemical and Biophysical Research Communications, vol. 268, no. 3, pp. 916-920, 2000.

[37] B. Drukarch and F. L. van Muiswinkel, "Neuroprotection for Parkinson's disease: a new approach for a new millennium," Expert Opinion on Investigational Drugs, vol. 10, no. 10, pp. 1855-1868, 2001.

[38] B. S. Mandavilli, S. F. Ali, and B. Van Houten, "DNA damage in brain mitochondria caused by aging and MPTP treatment," Brain Research, vol. 885, no. 1, pp. 45-52, 2000.

[39] H. Yamaguchi, K. Kajitani, Y. Dan et al., "MTH1, an oxidized purine nucleoside triphosphatase, protects the dopamine neurons from oxidative damage in nucleic acids caused by 1methyl-4-phenyl-1,2,3,6-tetrahydropyridine," Cell Death and Differentiation, vol. 13, no. 4, pp. 551-563, 2006.

[40] M. L. Selley, “(E)-4-Hydroxy-2-nonenal may be involved in the pathogenesis of Parkinson's disease," Free Radical Biology and Medicine, vol. 25, no. 2, pp. 169-174, 1998.

[41] L. I. P. Liang, J. Huang, R. Fulton, B. J. Day, and M. Patel, "An orally active catalytic metalloporphyrin protects against 1methyl-4-phenyl-1,2,3,6-tetrahydropyridine neurotoxicity in vivo," Journal of Neuroscience, vol. 27, no. 16, pp. 4326-4333, 2007. 
[42] M. S. Saporito, B. A. Thomas, and R. W. Scott, "MPTP activates c-Jun NH-terminal kinase (JNK) and its upstream regulatory kinase MKK4 in nigrostriatal neurons in vivo," Journal of Neurochemistry, vol. 75, no. 3, pp. 1200-1208, 2000.

[43] S. Karunakaran, U. Saeed, M. Mishra et al., "Selective activation of p38 mitogen-activated protein kinase in dopaminergic neurons of substantia nigra leads to nuclear translocation of p53 in 1-methyl-4-phenyl-1,2,3,6-tetrahydropyridine-treated mice," Journal of Neuroscience, vol. 28, no. 47, pp. 1250012509, 2008.

[44] S. Karunakaran and V. Ravindranath, "Activation of p38 MAPK in the substantia nigra leads to nuclear translocation of NF- $\kappa$ B in MPTP-treated mice: implication in Parkinson's disease," Journal of Neurochemistry, vol. 109, no. 6, pp. 17911799, 2009.

[45] M. Vila, V. Jackson-Lewis, S. Vukosavic et al., "Bax ablation prevents dopaminergic neurodegeneration in the 1-methyl4-phenyl-1,2,3,6-tetrahydropyridine mouse model of Parkinson's disease," Proceedings of the National Academy of Sciences of the United States of America, vol. 98, no. 5, pp. 2837-2842, 2001.

[46] H. Kamiya and H. Kasai, "Formation of 2-hydroxydeoxyadenosine triphosphate, an oxidatively damaged nucleotide, and its incorporation by DNA polymerases. Steady-state kinetics of the incorporation," The Journal of Biological Chemistry, vol. 270, no. 33, pp. 19446-19450, 1995.

[47] Y. Nakabeppu, D. Tsuchimoto, H. Yamaguchi, and K. Sakumi, "Oxidative damage in nucleic acids and Parkinson's disease," Journal of Neuroscience Research, vol. 85, no. 5, pp. 919-934, 2007.

[48] M. Ohno, S. Oka, and Y. Nakabeppu, "Quantitative analysis of oxidized guanine, 8-oxoguanine, in mitochondrial DNA by immunofluorescence method," Methods in Molecular Biology, vol. 554, pp. 199-212, 2009.

[49] S. Oka, M. Ohno, D. Tsuchimoto, K. Sakumi, M. Furuichi, and Y. Nakabeppu, "Two distinct pathways of cell death triggered by oxidative damage to nuclear and mitochondrial DNAs," The EMBO Journal, vol. 27, no. 2, pp. 421-432, 2008.

[50] M. L. Michaels, J. Tchou, A. P. Grollman, and J. H. Miller, "A repair system for 8-oxo-7,8-dihydrodeoxyguanine," Biochemistry, vol. 31, no. 45, pp. 10964-10968, 1992.

[51] S. Hirano, Y. Tominaga, A. Ichinoe et al., "Mutator phenotype of MUTYH-null mouse embryonic stem cells," The Journal of Biological Chemistry, vol. 278, no. 40, pp. 38121-38124, 2003.

[52] Y. Tominaga, Y. Ushijima, D. Tsuchimoto et al., "MUTYH prevents OGG1 or APEX1 from inappropriately processing its substrate or reaction product with its C-terminal domain," Nucleic Acids Research, vol. 32, no. 10, pp. 3198-3211, 2004.

[53] M. L. Michaels, J. Tchou, A. P. Grollman, and J. H. Miller, "A repair system for 8-oxo-7,8-dihydrodeoxyguanine," Biochemistry, vol. 31, no. 45, pp. 10964-10968, 1992.

[54] G. Simbula, P. A. Glascott Jr., S. Akita, J. B. Hoek, and J. L. Farber, "Two mechanisms by which ATP depletion potentiates induction of the mitochondrial permeability transition," American Journal of Physiology, vol. 273, no. 2, pp. C479-C488, 1997.

[55] P. S. Vosler, C. S. Brennan, and J. Chen, "Calpain-mediated signaling mechanisms in neuronal injury and neurodegeneration," Molecular Neurobiology, vol. 38, no. 1, pp. 78-100, 2008.

[56] S. J. Crocker, P. D. Smith, V. Jackson-Lewis et al., "Inhibition of calpains prevents neuronal and behavioral deficits in an MPTP mouse model of Parkinson's disease," Journal of Neuroscience, vol. 23, no. 10, pp. 4081-4091, 2003.
[57] T. Eki and J. Hurwitz, "Influence of poly(ADP-ribose) polymerase on the enzymatic synthesis of SV40 DNA," The Journal of Biological Chemistry, vol. 266, no. 5, pp. 3087-3100, 1991.

[58] C. Soldani and A. I. Scovassi, "Poly(ADP-ribose) polymerase1 cleavage during apoptosis: an update," Apoptosis, vol. 7, no. 4, pp. 321-328, 2002.

[59] S. W. Yu, H. Wang, M. F. Poitras et al., "Mediation of poty(ADP-ribose) polymerase-1-dependent cell death by apoptosis-inducing factor," Science, vol. 297, no. 5579, pp. 259-263, 2002.

[60] A. S. Mandir, S. Przedborski, V. Jackson-Lewis et al., "Poly(ADP-ribose) polymerase activation mediates 1-methyl4-phenyl-1,2,3,6-tetrahydropyridine (MPTP)-induced parkinsonism," Proceedings of the National Academy of Sciences of the United States of America, vol. 96, no. 10, pp. 5774-5779, 1999.

[61] H. Wang, M. Shimoji, S. W. Yu, T. M. Dawson, and V. L. Dawson, "Apoptosis inducing factor and PARP-mediated injury in the MPTP mouse model of Parkinson's disease," Annals of the New York Academy of Sciences, vol. 991, pp. 132139, 2003.

[62] S. Veto, P. Acs, J. Bauer et al., "Inhibiting poly(ADP-ribose) polymerase: a potential therapy against oligodendrocyte death," Brain, vol. 133, no. 3, pp. 822-834, 2010.

[63] C. C. Alano, W. Ying, and R. A. Swanson, "Poly(ADP-ribose) polymerase-1-mediated cell death in astrocytes requires NAD depletion and mitochondrial permeability transition," The Journal of Biological Chemistry, vol. 279, no. 18, pp. 1889518902, 2004.

[64] R. Heikkila and G. Cohen, "Inhibition of biogenic amine uptake by hydrogen peroxide: a mechanism for toxic effects of 6-hydroxydopamine," Science, vol. 172, no. 3989, pp. 12571258, 1971.

[65] G. Cohen and R. E. Heikkila, "The generation of hydrogen peroxide, superoxide radical, and hydroxyl radical by 6 hydroxydopamine, dialuric acid, and related cytotoxic agents," The Journal of Biological Chemistry, vol. 249, no. 8, pp. 24472452, 1974.

[66] D. Blum, S. Torch, N. Lambeng et al., "Molecular pathways involved in the neurotoxicity of 6-OHDA, dopamine and MPTP: contribution to the apoptotic theory in Parkinson's disease," Progress in Neurobiology, vol. 65, no. 2, pp. 135-172, 2001.

[67] A. Slivka and G. Cohen, "Hydroxyl radical attack on dopamine," The Journal of Biological Chemistry, vol. 260, no. 29, pp. 15466-15472, 1985.

[68] K. Jellinger, L. Linert, E. Kienzl, E. Herlinger, and M. B. H. Youdim, "Chemical evidence for 6-hydroxydopamine to be an endogenous toxic factor in the pathogenesis of Parkinson's disease," Journal of Neural Transmission, Supplement, no. 46, pp. 297-314, 1995.

[69] W. Linert, E. Herlinger, R. F. Jameson, E. Kienzl, K. Jellinger, and M. B. H. Youdim, "Dopamine, 6-hydroxydopamine, iron, and dioxygen - their mutual interactions and possible implication in the development of Parkinson's disease," Biochimica et Biophysica Acta, vol. 1316, no. 3, pp. 160-168, 1996.

[70] A. Palumbo, A. Napolitano, P. Barone, and M. D'Ischia, "Nitrite- and peroxide-dependent oxidation pathways of dopamine: 6- nitrodopamine and 6-hydroxydopamine formation as potential contributory mechanisms of oxidative stress- and nitric oxide-induced neurotoxicity in neuronal degeneration," Chemical Research in Toxicology, vol. 12, no. 12, pp. 1213-1222, 1999. 
[71] C. D. Garner and J. P. Nachtman, "Manganese catalyzed auto-oxidation of dopamine to 6-hydroxydopamine in vitro," Chemico-Biological Interactions, vol. 69, no. 4, pp. 345-351, 1989.

[72] G. R. Breese and T. D. Traylor, "Depletion of brain noradrenaline and dopamine by 6-hydroxydopamine," British Journal of Pharmacology, vol. 42, no. 1, pp. 88-99, 1971.

[73] F. Karoum, S. J. Chrapusta, M. F. Egan, and R. J. Wyatt, "Absence of 6-hydroxydopamine in the rat brain after treatment with stimulants and other dopaminergic agents: a mass fragmentographic study," Journal of Neurochemistry, vol. 61, no. 4, pp. 1369-1375, 1993.

[74] G. S. Kaminski Schierle, O. Hansson, E. Ferrando-May, P. Nicotera, P. Brundin, and M. Leist, "Neuronal death in nigral grafts in the absence of poly (ADP-ribose) polymerase activation," NeuroReport, vol. 10, no. 16, pp. 3347-3351, 1999.

[75] F. L. van Muiswinkel, B. Drukarch, H. W. M. Steinbusch, and J. De Vente, "Sustained pharmacological inhibition of nitric oxide synthase does not affect the survival of intrastriatal rat fetal mesencephalic transplants," Brain Research, vol. 792, no. 1, pp. 48-58, 1998.

[76] I. Ohsawa, M. Ishikawa, K. Takahashi et al., "Hydrogen acts as a therapeutic antioxidant by selectively reducing cytotoxic oxygen radicals," Nature Medicine, vol. 13, no. 6, pp. 688-694, 2007.

[77] K. Nagata, N. Nakashima-Kamimura, T. Mikami, I. Ohsawa, and S. Ohta, "Consumption of molecular hydrogen prevents the stress-induced impairments in hippocampus-dependent learning tasks during chronic physical restraint in mice," Neuropsychopharmacology, vol. 34, no. 2, pp. 501-508, 2009.

[78] Y. Gu, C. S. Huang, T. Inoue et al., "Drinking hydrogen water ameliorated cognitive impairment in senescence-accelerated mice," Journal of Clinical Biochemistry and Nutrition, vol. 46, no. 3, pp. 269-276, 2010.

[79] K. I. Fukuda, S. Asoh, M. Ishikawa, Y. Yamamoto, I. Ohsawa, and S. Ohta, "Inhalation of hydrogen gas suppresses hepatic injury caused by ischemia/reperfusion through reducing oxidative stress," Biochemical and Biophysical Research Communications, vol. 361, no. 3, pp. 670-674, 2007.

[80] B. M. Buchholz, D. J. Kaczorowski, R. Sugimoto et al., "Hydrogen inhalation ameliorates oxidative stress in transplantation induced intestinal graft injury," American Journal of Transplantation, vol. 8, no. 10, pp. 2015-2024, 2008.

[81] A. Nakao, D. J. Kaczorowski, Y. Wang et al., "Amelioration of rat cardiac cold ischemia/reperfusion injury with inhaled hydrogen or carbon monoxide, or both," Journal of Heart and Lung Transplantation, vol. 29, no. 5, pp. 544-553, 2010.

[82] K. Hayashida, M. Sano, I. Ohsawa et al., "Inhalation of hydrogen gas reduces infarct size in the rat model of myocardial ischemia-reperfusion injury," Biochemical and Biophysical Research Communications, vol. 373, no. 1, pp. 30-35, 2008.

[83] I. Ohsawa, K. Nishimaki, K. Yamagata, M. Ishikawa, and S. Ohta, "Consumption of hydrogen water prevents atherosclerosis in apolipoprotein E knockout mice," Biochemical and Biophysical Research Communications, vol. 377, no. 4, pp. 1195-1198, 2008.

[84] Y. Fu, M. Ito, Y. Fujita et al., "Molecular hydrogen is protective against 6-hydroxydopamine-induced nigrostriatal degeneration in a rat model of Parkinson's disease," Neuroscience Letters, vol. 453, no. 2, pp. 81-85, 2009.

[85] E. C. Stack, J. L. Ferro, J. Kim et al., "Therapeutic attenuation of mitochondrial dysfunction and oxidative stress in neurotoxin models of Parkinson's disease," Biochimica et Biophysica Acta, vol. 1782, no. 3, pp. 151-162, 2008.
[86] K. I. Setsukinai, Y. Urano, K. Kakinuma, H. J. Majima, and T. Nagano, "Development of novel fluorescence probes that can reliably detect reactive oxygen species and distinguish specific species," The Journal of Biological Chemistry, vol. 278, no. 5, pp. 3170-3175, 2003.

[87] Y. Sato, S. Kajiyama, A. Amano et al., "Hydrogen-rich pure water prevents superoxide formation in brain slices of vitamin C-depleted SMP30/GNL knockout mice," Biochemical and Biophysical Research Communications, vol. 375, no. 3, pp. 346350, 2008.

[88] A. Nakao, Y. Toyoda, P. Sharma, M. Evans, and N. Guthrie, "Effectiveness of hydrogen rich water on antioxidant status of subjects with potential metabolic syndrome-an open label pilot study," Journal of Clinical Biochemistry and Nutrition, vol. 46, no. 2, pp. 140-149, 2010.

[89] J. Alam and J. L. Cook, "How many transcription factors does it take to turn on the heme oxygenase-1 gene?" American Journal of Respiratory Cell and Molecular Biology, vol. 36, no. 2, pp. 166-174, 2007.

[90] D. T. Dexter, F. R. Wells, F. Agid et al., "Increased nigral iron content in postmortem Parkinsonian brain," The Lancet, vol. 2, no. 8569, pp. 1219-1220, 1987.

[91] A. Yoritaka, N. Hattori, K. Uchida, M. Tanaka, E. R. Stadtman, and Y. Mizuno, "Immunohistochemical detection of 4-hydroxynonenal protein adducts in Parkinson disease," Proceedings of the National Academy of Sciences of the United States of America, vol. 93, no. 7, pp. 2696-2701, 1996.

[92] Z. I. Alam, S. E. Daniel, A. J. Lees, D. C. Marsden, P. Jenner, and B. Halliwell, "A generalised increase in protein carbonyls in the brain in Parkinson's but not incidental Lewy body disease," Journal of Neurochemistry, vol. 69, no. 3, pp. 1326-1329, 1997.

[93] H. Shimura-Miura, N. Hattori, D. Kang, K. I. Miyako, Y. Nakabeppu, and Y. Mizuno, "Increased 8-oxo-dGTPase in the mitochondria of substantia nigral neurons in Parkinson's disease," Annals of Neurology, vol. 46, no. 6, pp. 920-924, 1999. 


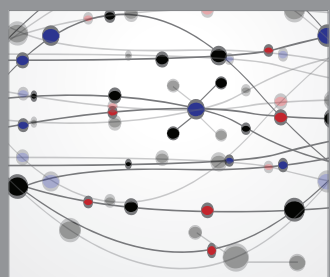

The Scientific World Journal
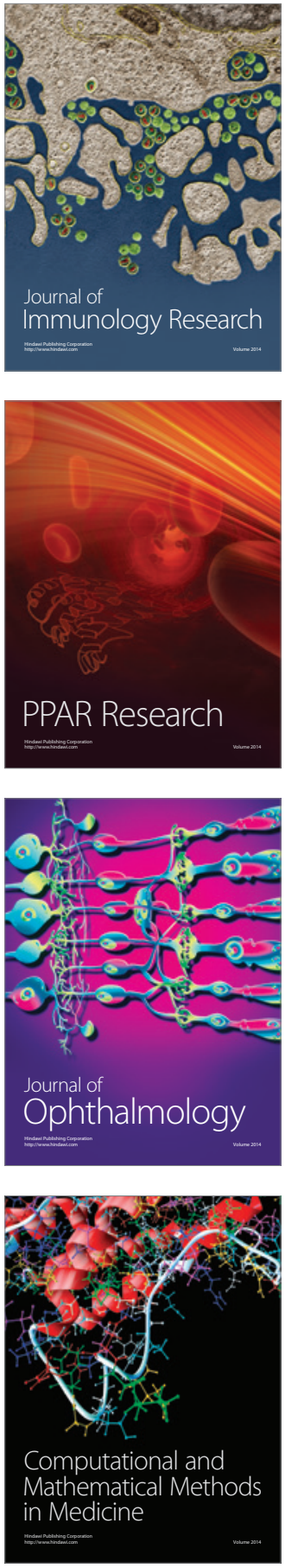

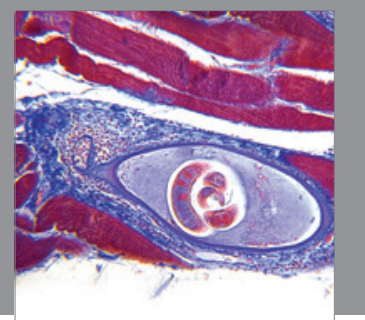

Gastroenterology

Research and Practice
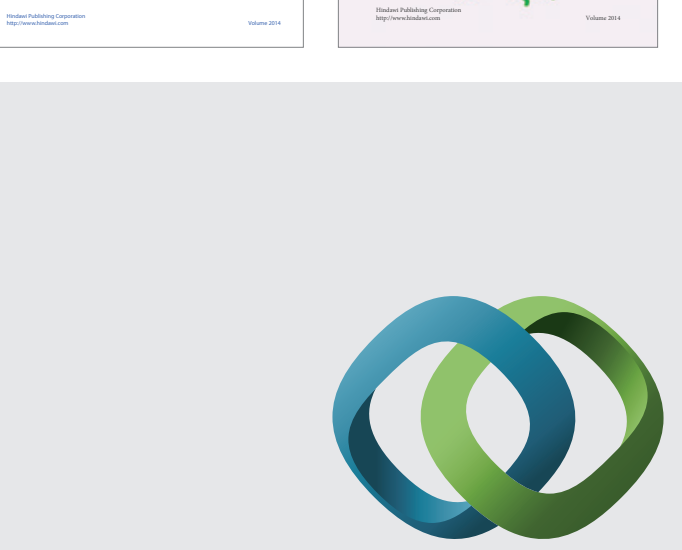

\section{Hindawi}

Submit your manuscripts at

http://www.hindawi.com
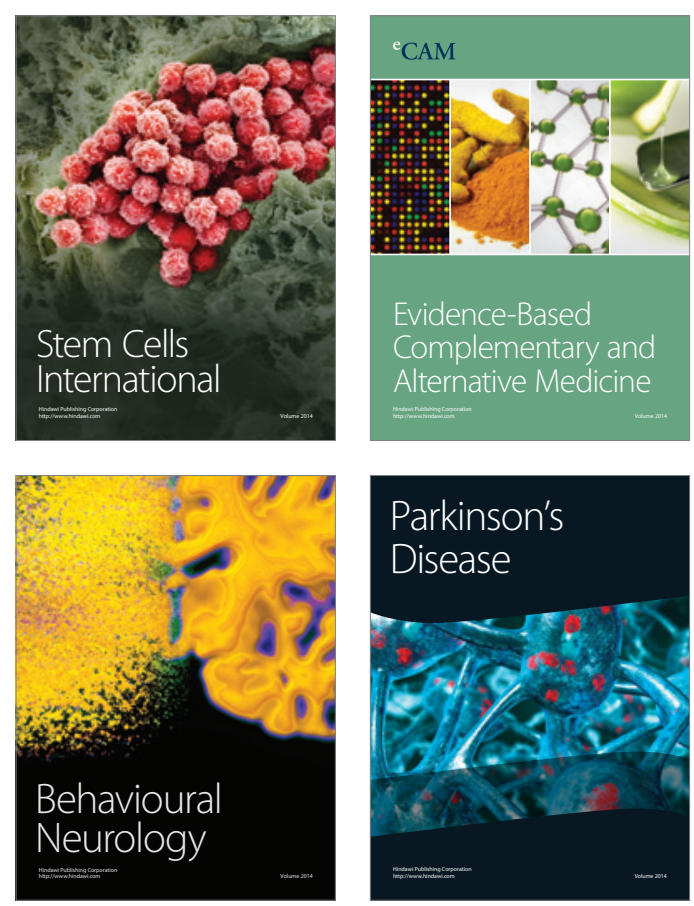

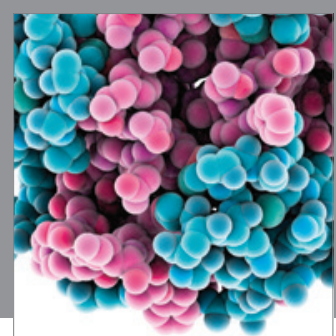

Journal of
Diabetes Research

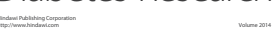

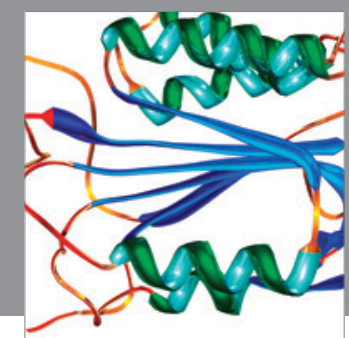

Disease Markers
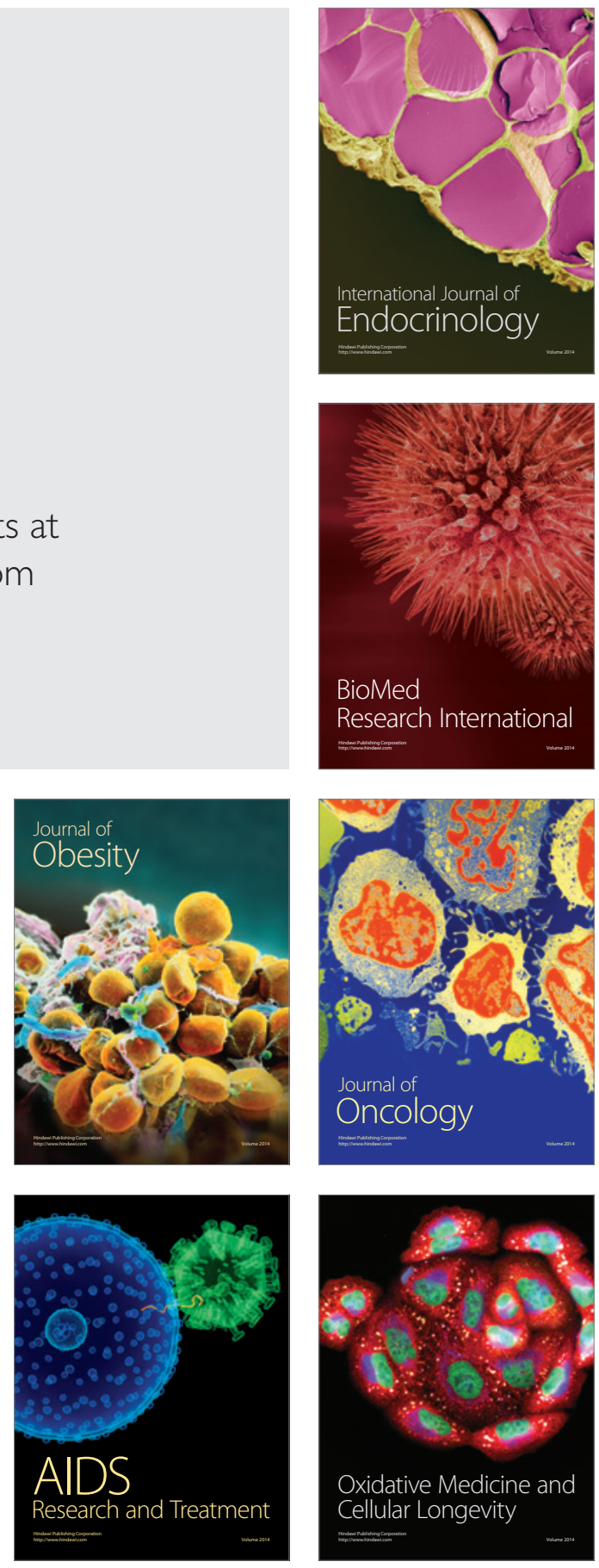